\title{
GLOBAL OSCILLATIONS IN Be STAR ENVELOPES:
}

\section{OBSERVATIONAL EVIDENCE}

\author{
R. W. HANUSCHIK ${ }^{*}$ \\ Astronomical Institute, University of Tübingen, D-72076 Tübingen, Germany \\ W. HUMMEL* \\ Astrofysisch Instituut, Vrije Universiteit Brussel, Pleinlaan 2, B-1050 Brussel, Belgium \\ and \\ O. DIETLE, J. DACHS and E. SUTORIUS \\ Astronomical Institute, Ruhr-Universität Bochum, D-44780 Bochum, Germany
}

\section{The observations}

Since 1982, we are performing a long-term spectroscopic observing programme of emission-lines in Be stars (Hanuschik 1987, Hanuschik et al. 1988, Dachs et al. 1992, Sutorius 1992, Dietle 1993). We are using ESO's $1.4 \mathrm{~m}$ CAT, at resolution $R \geq 50000$ and $S / N=100-1000$. Spectral lines chosen are the optically thick $\mathrm{H} \alpha, \mathrm{H} \beta$ lines and the optically thin Fe II $\lambda 5317$ line. The latter line is an extremely sharp tracer $\left(\Delta v_{\text {th }}=2 \mathrm{~km} \mathrm{~s}^{-1}\right)$ for the kinematics in the disks. We believe that our atlas shows the full range of intrinsic structure of these emission lines.

We find that the apparent wealth of line profile shapes can be ordered into a simple two-class scheme: symmetric lines, which are often double-peaked, in case of $\mathrm{H} \alpha$ often of winebottle-type shape (so-called class 1; see Fig. 1); and asymmetric lines, in case of Fe II often with a typical single-peaked, "steeple"-type profile shape (= class 2; Fig. 2). Shell lines are considered as special cases within the two main classes. The shape of class 1 profiles usually remains approximately constant, while class 2 profiles often show a typical $V / R<1 \Longrightarrow=1 \Longrightarrow>1$ variability pattern with cycles of the order of 7-10 years.

\section{The interpretation}

Taken together with other evidence (see Hanuschik, these proceedings), class 1 profiles are likely to occur in an axisymmetric disk-like envelope in steady state. The typical shape and variability pattern of class 2 profiles, however,

* On leave from: Astronomical Institute Bochum

** Affiliated to: Fachbereich Physik, Universität-GH Essen, D-45141 Essen, Germany 

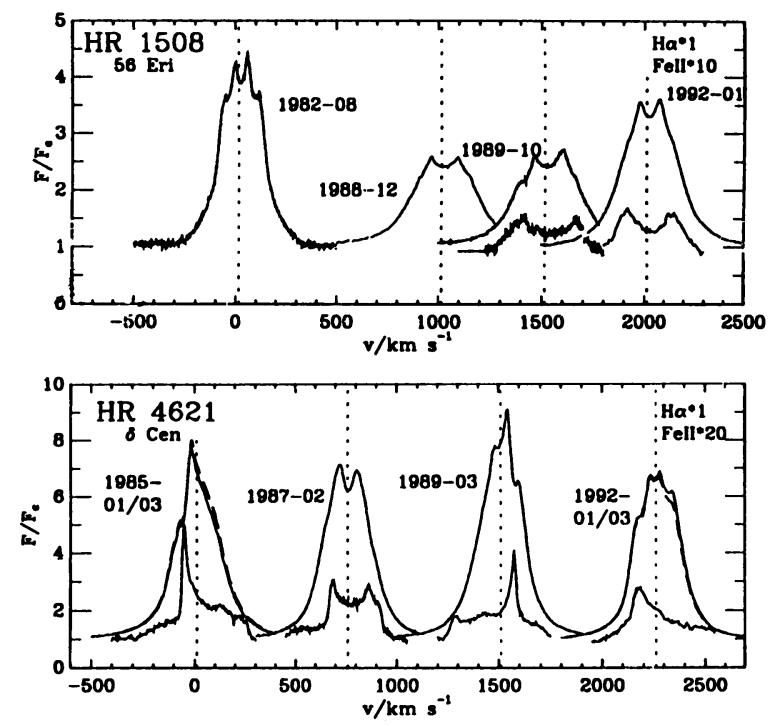

Fig. 1. Typical class 1 (top) and class 2 (bottom) line profiles. Broken lines indicate $\pm v \sin i$ and 0 in the stellar frame. Flux scale for Fe II line is enlarged by the factors given.

must be caused by a large-scale density inhomogeneity in the disk which is not co-rotating, but slowly precessing. We believe that such density perturbation is caused by a one-armed global oscillation as recently proposed by Okazaki (1991) and Papaloizou et al. (1992). As Hummel \& Hanuschik (these proceedings) show, model line profiles for such perturbation show similarity with the observed steeple-type class 2 profiles. As a consequence of the rotational flattening of the central star, the perturbation is expected to slowly precess, causing the long-term $V / R$ variations known since long (e.g., Doazan et al. 1987).

The origin of the density perturbation is unknown, but possibly related to outbursts on the star and thereby related to the mass ejection processes ultimately giving birth to the whole circumstellar disk.

\section{References}

Dachs J., Hummel W., Hanuschik R.W.: 1992, A\&AS 95, 437.

Dietle O.: 1993, Diploma thesis (Bochum).

Doazan V., Rusoni L., Sedmak G., Thomas R.N., Bourdonneau B.: 1987, A\&A 182, L25.

Hanuschik R.W.: 1987, $A \& A$ 173, 299.

Hanuschik R.W., Kozok J., Kaiser D.: 1988, $A \& A$ 189, 147.

Hummel W., Dachs J.: 1992, $A \& A$ 262, L17.

Okazaki T.: 1991, PASJ 43, 75.

Papaloizou J.C., Savonije G.J., Henrichs H.F.: 1992, $A \& A$ 265, L45.

Sutorius E.: 1992, Diploma thesis (Bochum). 